La Revue

des Droits

de l'Homme

\section{La Revue des droits de l'homme}

Revue du Centre de recherches et d'études sur les droits fondamentaux

$10 \mid 2016$

Revue des droits de l'homme - $\mathrm{N}^{\circ} 10$

\title{
Les récompenses financières des lanceurs d'alerte portent-elles atteinte aux droits fondamentaux? Le cas du droit américain
}

Johanna Schwartz Miralles

\section{(2) OpenEdition}

Édition électronique

URL : http://journals.openedition.org/revdh/2383

DOI : $10.4000 /$ revdh.2383

ISSN : 2264-119X

Éditeur

Centre de recherches et d'études sur les droits fondamentaux

Référence électronique

Johanna Schwartz Miralles, «Les récompenses financières des lanceurs d'alerte portent-elles atteinte aux droits fondamentaux ? Le cas du droit américain », La Revue des droits de l'homme [En ligne], 10 | 2016, mis en ligne le 12 juillet 2016, consulté le 22 juillet 2020. URL : http://journals.openedition.org/ revdh/2383; DOI : https://doi.org/10.4000/revdh.2383

Ce document a été généré automatiquement le 22 juillet 2020.

Tous droits réservés 


\title{
Les récompenses financières des lanceurs d'alerte portent-elles atteinte aux droits fondamentaux? Le cas du droit américain
}

\author{
Johanna Schwartz Miralles
}

1 La question des « lanceurs d'alerte et des droits de l'homme » posée par le colloque des 20 et 21 avril 2015 évoque, bien évidemment, la relation entre les droits fondamentaux et le lancement d'alerte. Dès lors, un bon nombre des interventions a mis l'accent sur la question - très pertinente, voire essentielle - des moyens permettant la protection du droit subjectif du lanceur d'alerte à la liberté d'expression. D'autres interventions ont posé la question, d'une égale importance, de la relation entre la protection du lanceur d'alerte et la promotion de la démocratie participative.

2 Je souhaiterais aborder l'étude de cette relation entre les droits fondamentaux et l'alerte d'une autre perspective - celle d'outre-Atlantique - en examinant le cas du lancement d'alerte en droit américain et en posant la question de savoir si les récompenses financières des lanceurs d'alerte, qui sont d'usage extrêmement courant aux États-Unis, permettent de promouvoir les droits fondamentaux, ou au contraire si elles portent atteinte à ces mêmes droits.

3 Les lois américaines relatives au lancement d'alerte sont parmi les plus anciennes au monde ${ }^{1}$. Elles sont abondantes, abondance qui résulte de la structure fédérale du pays et de son système de Common Law, qui permet aux juges aussi bien qu'aux législateurs fédéraux et étatiques de façonner la protection des lanceurs d'alerte ${ }^{2}$.

4 Le droit d'alerte américain tire également sa particularité du rôle qui y est joué par les récompenses financières. Divers dispositifs de droit américain tels que le False Claims Act $^{3}$, le Dodd-Frank Wall Street Reform and Consumer Protection Act ${ }^{4}$, ou la IRS Whistleblower Law ${ }^{5}$ incitent les lanceurs d'alerte à fournir des informations sur des actes illégaux dont ils ont pu être témoins au sein de leur entreprise ou sur leur lieux de travail. Les sommes versées aux lanceurs d'alerte sous l'égide de ces lois peuvent être 
considérables: certains lanceurs d'alerte ont déjà obtenu des primes s'élevant à plusieurs millions, voire des centaines de millions de dollars.

5 Les États-Unis n'est pas le seul pays qui prévoit des récompenses pour certains lanceurs d'alerte ${ }^{6}$. Pourtant, c'est le pays où cette pratique est la plus répandue. La France, pour sa part, s'avère actuellement très réticente quant à la rémunération des lanceurs d'alerte ${ }^{7}$. Parmi les raisons pour cette réticence, le législateur français estime que rémunérer le lanceur d'alerte peut provoquer des alertes abusives qui nuisent aux droits des personnes visées dans l'alerte ${ }^{8}$.

Du fait du rôle singulier des récompenses prévues par la loi américaine, analyser cette loi nous permet d'examiner les éventuels conflits entre, d'une part, la protection des droits des lanceurs d'alerte et la promotion de l'intérêt général, et d'autre part, la protection des droits fondamentaux des lanceurs d'alerte, du public, et des personnes et entreprises mises en cause par l'alerte. Dès lors, il convient d'examiner les principaux dispositifs d'alerte en droit américain qui prévoient des récompenses pour les lanceurs d'alerte (I). Il convient ensuite de considérer certains avantages et inconvénients de ces récompenses, notamment quant à la protection des droits fondamentaux tels que le droit à la vie, à la santé, ou à un environnement sain (II).

\section{I - Les dispositifs d'alerte américains prévoyant des récompenses financières}

7 De nombreuses lois américaines récompensent les lanceurs d'alerte, en plus d'interdire des mesures de représailles à leur égard. Ces lois sont nombreuses, mais en général, il est possible de les classer en deux catégories: des lois qui tam (A) et des lois compensatoires stricto sensu (B).

\section{A - Les lois qui tam}

8 Certaines lois américaines, dites des lois qui $\operatorname{tam}^{9}$, donnent au lanceur d'alerte le droit d'agir en justice, au nom du gouvernement, contre une personne physique ou morale que le lanceur d'alerte accuse d'avoir commis des actes frauduleux ou illégaux. Le lanceur d'alerte est autorisé non seulement à porter plainte au nom de l'État et dans l'intérêt public, mais également à partager avec l'État les éventuelles sommes récupérées.

Dans une action qui tam, le lanceur d'alerte fait prévaloir non pas ses propres droits, mais ceux de l'État. Une récompense financière lui est accordée au motif qu'il a apporté des informations essentielles à la connaissance du public, ainsi qu'en reconnaissance de ses efforts au cours du procès.

De manière générale, une loi qui tam contient un aspect «substantiel» et un aspect "procédural». Quant à la partie «substantielle», il s'agit d'une interdiction - par exemple, de frauder le gouvernement ${ }^{10}$, de piller une épave ${ }^{11}$, ou de vendre des boissons alcoolisées $^{12}$. Dans ce sens, une loi qui tam ressemble en tout point à une loi classique qui prohibe des actes que l'on estime porter atteinte à l'intérêt général.

11 La singularité des lois qui tam se trouve non pas dans leur aspect substantiel, mais plutôt dans leur aspect procédural, qui, comme indiqué ci-dessus, voudrait que les lanceurs d'alerte intentent une action en justice au nom du gouvernement. La loi qui 
tam promet au lanceur d'alerte, devenu partie demanderesse, une partie de la somme récupérée.

12 L'exemple typique d'une loi qui tam est le False Claims Act, une loi fédérale qui date de l'année 1863. Le Congrès américain a voté cette loi au cours de la guerre de Sécession afin de faire face à des fraudes perpétrées par des fournisseurs du gouvernement ${ }^{13}$. Des fournisseurs peu scrupuleux auraient, en effet, vendu aux armées de l'Union des marchandises défectueuses comme, par exemple, de la poudre coupée à la sciure de bois $^{14}$. Face à de nombreuses ventes fallacieuses mais difficiles à déceler, le Congrès estima que la meilleure manière de confondre les malfaiteurs serait d'obtenir des renseignements de la part des personnes ayant une connaissance intime des fraudes ${ }^{15}$.

13 La loi que le Congrès a, par la suite, votée - qui a été modifiée à plusieurs reprises depuis sa première publication en 1863 - proscrit toute fraude liée aux déboursements du gouvernement fédéral ${ }^{16}$ et permet aux lanceurs d'alerte d'intenter une action en justice au nom du gouvernement contre le fraudeur présumé.

14 La mise en œuvre du False Claims Act est assez singulière, ce qui rend son analyse d'autant plus intéressante. Pour intenter une action qui tam, le lanceur d'alerte ${ }^{17}$ porte plainte dans un tribunal fédéral de première instance (United States district court) ${ }^{18}$. La plainte est déposée et gardée sous scellés de sorte que ni l'accusé, ni le public ne soit informé de son existence ${ }^{19}$. Une fois la plainte déposée, le plaignant doit en transmettre une copie au procureur général des États-Unis (Attorney General). Le plaignant fournit également au procureur tout élément de preuve sur lequel la plainte est fondée ${ }^{20}$. Le ministère de la Justice (Department of Justice) jouit, par la suite, d'un délai de 60 jours après réception de la plainte pour investiguer son bien-fondée ${ }^{1}$. À la fin de cette période, le procureur général doit prendre la décision d'intervenir ou non aux poursuites («intervene »). Si le procureur général choisit d'intervenir, il lui revient de diriger l'enquête et les poursuites. Si le procureur décide, en revanche, de ne pas intervenir, il reste au lanceur d'alerte la possibilité de poursuivre l'action à titre personnel ${ }^{22}$.

15 Il est important de souligner que, jusqu'à ce que le procureur opte, ou non, pour la participation, la partie défenderesse n'a pas connaissance de l'existence des poursuites entamées. La plainte n'est, en effet, rendue publique et notifiée à l'accusé qu'au moment où le procureur informe le tribunal de sa décision quant à sa participation aux poursuites.

Le lanceur d'alerte qui a gain de cause ou qui conclut un accord transactionnel avec la partie défenderesse partage, avec l'État, les dommages-intérêts. De manière générale, si le procureur général n'a pas participé au procès, le lanceur d'alerte se verra allouer entre 25 et 30 pour cent du montant de la condamnation rendue au titre du jugement ou du règlement, contre entre 15 et 25 pour cent si le procureur est intervenu au procès ${ }^{23}$. Cependant, ces pourcentages en tant que tels ne permettent pas de saisir l'importance des primes. Il faut, en effet, pour cela savoir quels sont les montants totaux des jugements et règlements. Ces sommes sont conséquentes, car le False Claims Act condamne la partie perdante à verser, en sus d'importantes pénalités règlementaires, trois fois le montant des dommages réels subis par le gouvernement ( treble damages »). Ces pénalités et dommages s'accumulent vite.

Pour ne citer que quelques cas concrets, en 2009, Pfizer, le géant du domaine pharmaceutique, conclut un accord à l'amiable avec le gouvernement américain prévoyant le paiement d'une pénalité d'un montant de 2,3 milliards de dollars. Cent 
deux millions de dollars de cette pénalité furent octroyés aux six donneurs d'alerte ${ }^{24}$. En 2012, une autre société pharmaceutique, GlaxoSmithKline, a versé aux États-Unis une pénalité d'un montant de 3 milliards de dollars ${ }^{25}$; le principal lanceur d'alerte en reçut 96 millions ${ }^{26}$.

$\mathrm{Au}$ vu des montants importants versés dans ces litiges, il n'est pas surprenant d'apprendre que la loi se révèle efficace ${ }^{27}$. Au cours de l'année 2014, les États-Unis ont ainsi recouvré environ 5,69 milliards de dollars grâce au False Claims Act; entre les années 2009 et 2014, le montant s'est élevé à 22,75 milliards de dollars ${ }^{28}$. Une étude de 2013 a estimé que chaque dollar dépensé dans un procès de type «False Claims Act » en rapporte vingt ${ }^{29}$.

Le False Claims Act n'est pas la seule loi qui tam qui existe aux États-Unis. Au niveau fédéral, deux autres lois, le Patent $A c t^{30}$ et une loi sur la protection des peuples indigènes ${ }^{31}$, prévoient des primes par voie d'une action qui tam ${ }^{32}$. De nombreux états fédérés et municipalités ont également adopté des false claims acts qui s'appliquent aux fraudes liées aux déboursements des gouvernements étatiques et municipaux ${ }^{33}$. Le False Claims Act fédéral est pourtant celui qui est le plus souvent invoqué et qui contribue le plus à la rémunération des lanceurs d'alerte.

\section{B - Les lois compensatoires stricto sensu}

20 Outre des lois qui tam, de nombreux dispositifs américains prévoient des primes pour les lanceurs d'alerte, sans pour autant leur permettre d'ester en justice ${ }^{34}$. Ces lois disposent que le gouvernement - et non le lanceur d'alerte - mène l'enquête et l'éventuel procès; le rôle du lanceur d'alerte s'achève au moment où il a communiqué aux autorités publiques tout renseignement utile. Il ne lui reste alors qu'à attendre sa récompense.

21 Parmi ces lois compensatrices, la plus récente est la Dodd-Frank Act, votée par le Congrès américain en 2010. Cette loi autorise la U.S. Securities and Exchange Commission ( $S E C$ »), l'autorité américaine des marchés financiers, à octroyer des primes aux lanceurs d'alerte lorsqu'ils fournissent des informations permettant au gouvernement de recouvrer plus de 1 million de dollars ${ }^{35}$. Le lanceur d'alerte perçoit entre 10 et 30 pour cent de la somme recouvrée ${ }^{36}$. La loi prévoit également que l'identité du lanceur demeurera confidentielle ${ }^{37}$ et interdit toutes représailles à son égard ${ }^{38}$.

Entre 2011 et 2015, la Securities and Exchange Commission a récompensé plus de vingt lanceurs d'alerte ${ }^{39}$. La plus grande prime versée jusqu'à présent s'élève à 30 millions de dollars ${ }^{40}$.

L'application de la loi Dodd-Frank soulève la question de son extraterritorialité, car la prime prévue par cette loi n'est pas soumise à des conditions liées à la nationalité du lanceur d'alerte, ni à son lieu de travail. Un lanceur d'alerte étranger travaillant hors des États-Unis peut, en conséquence, bénéficier du dispositif dès lors qu'il fournit des informations relatives au non-respect des lois financières américaines ${ }^{41}$.

Dans l'ensemble, la présidente de la SEC M.J. White estime que le bilan est positif ${ }^{42}$ : au cours de l'année 2015, la SEC a été contactée à plus de 4.000 reprises par les lanceurs d'alerte et, dans plusieurs cas, les informations fournies ont conduit à des enquêtes et des poursuites pour violation de la loi de finance ${ }^{43}$. 
D'autres lois américaines fonctionnent de manière semblable à la loi Dodd-Frank. Un exemple pertinent est celui de l'IRS Whistleblower Law, qui promet des primes aux aviseurs fiscaux ${ }^{44}$. C'est en vertu de cette loi que Bradley Birkenfeld, ancien salarié de la banque suisse UBS, a pu percevoir en 2012 une prime s'élevant à 104 millions de dollars en échange des informations qu'il a fournies aux autorités publiques relatives à l'évasion fiscale de la banque et de ses clients ${ }^{45}$.

\section{II - Les avantages et inconvénients des récompenses financières}

La loi américaine propose souvent des récompenses financières afin d'encourager l'alerte. Si ces lois existent en abondance, l'on pourrait imaginer qu'elles présentent des avantages - peut-être, même, des avantages liés à la protection des droits fondamentaux (A). Cependant, si l'abondance de ces dispositifs nous amène à négliger l'examen des risques qu'ils entraînent (B), l'on aura commis l'erreur de raisonnement de Pangloss ${ }^{46}$, qui partant de la réalité positive en tire une conclusion normative. Pour ne pas tomber dans cette erreur de raisonnement, il convient de poser la question des avantages et des inconvénients des récompenses financières des lanceurs d'alerte.

\section{A - Une protection accrue des droits fondamentaux?}

De nombreux ouvrages et articles traitent des avantages des lois qui récompensent les lanceurs d'alerte ${ }^{47}$. Quelques points soulevés par ces articles semblent particulièrement pertinents sur la question de l'interaction entre les récompenses financières et les droits fondamentaux.

Dans un premier temps, les récompenses financières encouragent l'alerte ${ }^{48}$, ce qui peut promouvoir la protection des droits fondamentaux. Il arrive fréquemment qu'un salarié détienne des informations dont la révélation est essentielle à la protection de la vie, la santé, l'environnement ou l'économie ${ }^{49}$. Les « insiders » ont ainsi révélé des défauts de conception sur des voitures Ford Pinto dans les années 1970 qui ont provoqué des centaines de décès et de blessures, ou des manquements aux règles de sécurité aux installations nucléaires méconnus des régulateurs ${ }^{50}$.

Certains secteurs présentent un risque élevé. L'on pourrait prendre comme exemple les secteurs pharmaceutique, financier, ou énergétique, qui représentent une part croissante de l'activité économique de pays tels la France ou les États-Unis. Dans ces secteurs, les risques pour la santé publique, l'environnement, ou la sécurité économique sont omniprésents. Par ailleurs, le régulateur national peut être confronté à des difficultés liées à l'évaluation de la sécurité des produits et des procédés de fabrication, notamment du fait de son manque d'accès, de ressources, et de connaissances techniques à la hauteur de la complexité et de la vitesse des évolutions technologiques ${ }^{51}$.

Les salariés, en revanche, jouissent d'une connaissance intime des produits et procédés de fabrication. Ils possèdent des compétences techniques et une connaissance des processus de fabrication qui leur permettent de déceler les risques plus facilement et plus tôt que les régulateurs. Si les récompenses financières les incitent à révéler les risques et les dangers, au lieu de les dissimuler ou, tout simplement, de les ignorer, ces 
récompenses auront servi à protéger les droits fondamentaux tels que le droit à un environnement sain, à la santé, ou à la vie, ainsi que, dans le secteur financier, tous les droits fondamentaux qui peuvent exister dans une économie robuste (droit à l'éducation, à l'emploi, à l'accès à la culture). Promouvoir l'alerte par le moyen des récompenses peut, donc, avoir un effet positif non-négligeable sur les droits fondamentaux.

31 Par ailleurs, les récompenses financières augmentent la probabilité du traitement de l'alerte, ce qui a également des conséquences sur le respect des droits fondamentaux. Dans le cas d'une loi qui tam, le lien entre la récompense financière et le traitement de l'alerte est simple à définir. C'est en effet le lanceur d'alerte lui-même qui poursuit l'action, déposant plainte auprès des instances judiciaires et veillant, avec son avocat (et parfois aux côtés du procureur général), au bon déroulement du procès. Impliqué dans chaque étape du procès, le lanceur d'alerte peut assurer que son grief ne soit pas négligé.

Dans le cas des récompenses octroyées par les agences administratives, le lien entre la récompense financière et le traitement de l'alerte est moins direct, mais néanmoins présent. Il y a des raisons structurelles de craindre la faillite des agences administratives quant au traitement de l'alerte: une allocation de ressources financières et humaines insuffisantes, des règles internes complexes et lourdes qui sont des freins à l'action. Dans certains cas, l'explication est moins anodine: des conflits d'intérêts et des faits de corruption qui conduisent l'agence à favoriser les entités règlementées au détriment des donneurs d'alerte ${ }^{52}$.

33 En réalité, les antécédents ne sont pas prometteurs. Les agences administratives américaines telles que l'Occupational Safety and Health Administration, le IRS Whistleblower Office, ou le Merit Systems Protection Board n'ont pas toujours réussi à traiter les alertes de manière ponctuelle et impartiale ${ }^{53}$. Il semble que le cas français est similaire ${ }^{54}$.

La rémunération des lanceurs d'alerte sert-elle à atténuer ce problème d'inaction de la part des agences administratives ? Peut-être. Logiquement, le lanceur d'alerte (ou son avocat) qui attend une récompense aura tendance à suivre l'alerte auprès de l'agence où il l'a remise, demandant périodiquement des renseignements sur le traitement de son alerte. Plus les informations du lanceur d'alerte sont fiables et les enjeux de l'alerte importants, plus le lanceur d'alerte aura tendance à vouloir suivre son alerte, car il peut s'attendre à une récompense plus grande et plus certaine. Ce suivi de la part du lanceur d'alerte ou son avocat peut conduire une agence à mieux traiter l'alerte.

C'est un point essentiel pour deux raisons. D'une part, une alerte non traitée est une alerte dénuée de toute puissance. Pour protéger le public, le régulateur doit agir face à l'alerte, ou du moins, face aux alertes bien fondées et portant sur des questions d'intérêt général. Si les récompenses financières augmentent la probabilité des agissements de la part des agences publiques, elles auront protégé les droits fondamentaux qui sont mis en péril par le comportement qui a suscité l'alerte.

D'autre part, le traitement efficace de l'alerte peut inciter aux alertes. Car les lanceurs d'alerte sont, dans la plupart des cas, désireux de voir leurs alertes prises au sérieux. Ils espèrent, avant tout, mettre fin à la situation d'illégalité ou de danger qu'ils signalent ${ }^{55}$. S'ils pensent que l'alerte tombera dans un vide administratif, ils seront moins susceptibles, ceteris paribus, de la lancer. Si l'alerte porte sur les droits fondamentaux, 
un système qui promeut l'alerte en assurant qu'elle sera traitée est de loin préférable à un système qui la néglige.

Enfin, récompenser les lanceurs d'alerte s'avère efficace du point de vue des finances publiques. Comme indiqué plus haut, l'État américain a recouvré plusieurs milliards de dollars grâce aux actions qui tam ${ }^{56}$. À cette somme s'ajoutent environ 180 et 540 millions de dollars supplémentaires déjà recouvrés en vertu du Dodd Frank Act ${ }^{57}$. Même ceux qui, pour des raisons variées, s'opposent à ces lois, admettent qu'elles ont permis à l'État de recouvrer des sommes immenses ${ }^{58}$. En outre, les récompenses financières ont un effet économique en amont grâce à leur effet dissuasif sur les malfaiteurs potentiels. Car si les récompenses financières augmentent la probabilité qu'un lanceur d'alerte fournira des informations aux régulateurs, les entreprises seront moins susceptible de violer la loi $^{59}$. Dans le cas des procès qui tam, il y a un avantage financier supplémentaire : Le lanceur d'alerte, et surtout son avocat, prennent en charge une grande partie des coûts liés à l'enquête et la poursuite de l'action en justice, ce qui diminue les dépenses publiques qui auraient pu être occasionnées autrement ${ }^{60}$.

Le fait que les récompenses pour les lanceurs d'alerte permettent au gouvernement de faire des économies et de recouvrir des fonds peut avoir, comme effet indirect, la protection des droits fondamentaux des citoyens. Or, si le rôle du gouvernement est de promouvoir le bien public et de garantir les droits fondamentaux des citoyens par la voie des dépenses publiques, protéger les finances du gouvernement ne revient-il pas également protéger ses fins?

\section{B - Des atteintes aux droits fondamentaux?}

Le législateur français hésite à implémenter les incitations financières pour les lanceurs d'alerte ${ }^{61}$. Cette hésitation est-elle due - implicitement, si ce n'est pas explicitement aux effets que ces récompenses peuvent avoir sur des droits fondamentaux? Il conviendrait de se poser cette question en examinant les effets des récompenses financières sur les droits fondamentaux des personnes physiques mises en cause par l'alerte, ainsi que sur les droits des tiers et ceux des lanceurs d'alerte eux-mêmes.

Dans un premier temps, il faut s'interroger sur les droits fondamentaux des personnes mises en cause par l'alerte. Les instances françaises et européennes ont rappelé à plusieurs reprises la nécessité de protéger non seulement les lanceurs d'alerte mais également les accusés ${ }^{62}$. Ils ont exprimé ce souci devant les dispositifs qui ne visaient qu'à la protection du lanceur d'alerte, sans lui offrir de quelconque rémunération. Si l'alerte, en elle-même, risque de nuire aux droits des personnes mises en cause, la récompense financière pour cette alerte ne le fait-elle pas d'avantage?

Le problème principal est celui des alertes abusives ${ }^{63}$. Se défendre contre des accusations d'illégalité - que ce soit en justice ou lors d'une enquête administrative engendre des coûts importants, autant financiers que personnels et émotionnels. Selon une étude de 1998, une entreprise mise en cause dans une action qui tam déboursait, à l'époque où l'étude avait été faite, entre 250000 et 10000000 dollars pour ses frais de défense ${ }^{64}$. D'importants frais et pertes sont également été occasionnés en temps et en ressources perdus lors de l'enquête interne et de la collecte des matériels pour la défense ${ }^{65}$. À tout cela, s'ajoute la gêne d'être mis en cause, ainsi que les effets pervers qu'il peut y avoir sur la réputation de la personne impliquée par l'alerte, laquelle peut souffrir même si la personne est finalement acquittée. 

récompensant les lanceurs d'alertes légitimes. Il y a, tant en droit français qu'américain, la possibilité de poursuivre une action contre le lanceur d'alerte, par exemple une action en diffamation ou pour dénonciation calomnieuse ${ }^{66}$. Il y a également la possibilité de faire payer au lanceur d'alerte les frais de justice occasionnés par la victime ${ }^{67}$. Mais il peut néanmoins s'avérer difficile de réparer pleinement le dommage causé à la victime par une alerte abusive, car les préjudices sont souvent immatériels et donc difficiles à cerner et à prouver.

En plus d'inciter les alertes abusives, l'on peut craindre que la possibilité de "gagner gros» encourage d'autres comportements illégaux de la part des lanceurs d'alerte comme, par exemple, le vol de documents ou autres informations de nature confidentielle ou secrète - au détriment des droits des cibles de l'alerte ou des tiers. L'arrêt Cafasso v. General Dynamics ${ }^{68}$ présente un cas assez typique de ce type de comportement. Dynamics, a intenté une action qui tam alléguant que General Dynamics avait fraudé l'État en manquant à une obligation contractuelle d'informer ce dernier de l'existence de certaines nouvelles technologies qu'il avait, selon elle, le droit d'exploiter. Afin d'appuyer sa demande, la lanceuse d'alerte avait copié, sans autorisation, des dizaines de milliers de fichiers électroniques, fichiers qu'elle a par la suite communiqués à son avocat.

L'entreprise a formulé, en réponse, une demande reconventionnelle pour rupture de contrat, au motif que Mme Cafasso avait signé un accord interdisant toute divulgation d'information confidentielle de l'entreprise. En réponse, Mme Cafasso a fait valoir qu'elle avait pris les documents dans le but d'établir la fraude. Selon elle, appliquer la clause de confidentialité heurterait l'ordre public en empêchant des lanceurs d'alerte de fournir des preuves et constituerait donc une entrave à l'alerte.

La cour a estimé qu'en principe, interdire à un lanceur d'alerte de partager des preuves d'illégalité pourrait heurter l'ordre public. La cour a cependant jugé que, pour que les exigences de l'ordre public prévalent sur des obligations contractuelles de confidentialité, le lanceur d'alerte devait établir que « l'acte de prendre les documents était raisonnablement nécessaire à la poursuite de l'action ${ }^{69}$ ".

En l'espèce, la lanceuse d'alerte n'a pas pu établir cette nécessité. En copiant les documents, la lanceuse d'alerte avait téléchargé des dossiers entiers sans regarder si les documents individuels qu'ils contenaient présentaient un intérêt pour l'affaire. De plus, certains documents copiés étaient particulièrement sensibles, comme «des communications soumises au secret professionnel, des secrets commerciaux de [General Dynamics] et d'autres sociétés, des documents révélant des informations sur la recherche et le développement, des informations sensibles du gouvernement, et une demande de brevet qui bénéficiait d'une ordonnance de confidentialité de la part de l'office de Brevets américain ${ }^{70} »$.

48 Cet arrêt souligne les dangers liés à la sur-incitation à l'alerte. Ces dangers peuvent être encore plus importants si les documents pris par le lanceur d'alerte contiennent des données personnelles ou confidentielles des salariés ou des tiers ${ }^{71}$. Dans un dossier récent dans le secteur de l'éducation, par exemple, un ex-salarié avait pris des milliers de documents contentant des données personnelles d'étudiants (noms, adresses,

La Revue des droits de l'homme, 10 | 2016 
numéros de sécurité sociale, résultats scolaires, etc.). Ces étudiants n'étaient pas, euxmêmes, impliqués dans l'affaire, mais le lanceur d'alerte considérait que ces documents étaient suffisamment pertinents pour caractériser une fraude.

La réponse des tribunaux américains - de mettre en balance, au cas par cas, les intérêts de l'entreprise et des tiers, et la nécessité du lanceur d'alerte de divulguer les informations - est insuffisante, car bien souvent, les personnes tierces ne sont pas présentes lors du litige pour faire prévaloir leurs droits; parfois, elles ne sont même pas informées du fait que le lanceur d'alerte est en possession de leurs données personnelles. Un lanceur d'alerte, comme celui de l'affaire Cafasso, qui attend une grosse prime peut donc être tenté d'agir d'une manière qui nuit aux droits de son employeur ou des tiers, sans qu'il existe des garde-fous suffisants.

50 Il est également légitime de poser la question de l'effet des récompenses financières sur le traitement de l'alerte. S'agissant des alertes faites auprès des autorités publiques, il faut poser la question de savoir si les récompenses financières sur-incitent à l'alerte, et surtout aux alertes infondées, avec le résultat que les agences publiques chargées de traiter les alertes deviennent submergées et incapables de faire le tri entre les alertes importantes et celles qui manquent de légitimité. Dans ce cas, les récompenses financières auraient eu, pour effet, la diminution du traitement des alertes légitimes, avec une égale diminution de l'efficacité de la protection des droits des tiers impliqués par les alertes non-traitées. Ce problème nous semble moins critique s'il existe un mécanisme comme celui du qui tam permettant au lanceur d'alerte lui-même de poursuivre son action. En revanche, si le traitement de l'alerte est uniquement effectué par les agences publiques, la question de la surabondance des alertes se pose pleinement.

51 Il faut aussi se demander dans quelle mesure les récompenses financières affaiblissent les voies internes de traitement de l'alerte ${ }^{72}$. Ces voies internes peuvent être bien définies et officielles (des hotlines, par exemple) ou plus informelles (une conversation dans un bureau avec son supérieur hiérarchique). Les voies internes sont souvent plus efficaces que les voies externes pour mettre fin à des comportements nuisibles. Une alerte interne peut provoquer, par exemple, des mesures correctrices rapides et efficaces de la part de l'entreprise ${ }^{73}$, surtout si l'alerte concerne un comportement qui va à l'encontre des intérêts de l'entreprise ou dont la direction n'avait pas conscience ${ }^{74}$. $\mathrm{Du}$ point de vue éthique, il semblerait également juste de vouloir permettre à l'entreprise de corriger des problèmes internes dont elle n'avait pas conscience, avant de souffrir d'une publicité négative ou de subir des contrôles. En principe, il est donc préférable qu'un salarié lance une alerte en interne avant de se diriger vers les autorités publiques ou les médias. Les récompenses financières peuvent inciter les lanceurs d'alerte à se diriger directement vers des autorités publiques au lieu de passer par les voies internes.

52 Enfin, serait-il possible d'envisager que la rémunération d'un lanceur d'alerte puisse nuire aux droits fondamentaux du lanceur d'alerte ou des tiers en ébranlant la confiance du public dans le bien-fondé de l'alerte ? Pour exercer pleinement sa liberté d'expression, le lanceur d'alerte doit non seulement parler, mais aussi être entendu et écouté. Dans la mesure où son alerte met en jeu les droits fondamentaux des victimes potentielles de l'acte dénoncé, les droits fondamentaux des tiers et du public sont également impliqués. Est-ce le fait que le lanceur d'alerte qui perçoit un gain financier lui ôte sa fiabilité auprès de ses interlocuteurs? Certains l'ont suggéré ${ }^{75}$. Or, celui ou 
celle qui est rémunérée pour son alerte n'est plus motivé uniquement par l'intérêt général ; l'apparence d'impartialité, sinon l'impartialité elle-même, est compromise.

Y a-t-il, comme conséquence, une atteinte à la liberté d'expression du lanceur d'alerte, dans la mesure où il perd le droit de s'exprimer et d'être entendu par des auditeurs impartiaux? Il faut conclure que non. Le lanceur d'alerte désirant dissiper tout soupçon de mauvaise foi ou d'impartialité à son égard reste libre de refuser la récompense financière prévue par la loi ou d'en faire don, par exemple, à une association à but caritatif. Le choix de prendre l'argent ou non reste celui du lanceur d'alerte, qui assume les conséquences de son choix devant les a priori de ses éventuels détracteurs.

Quid des droits fondamentaux des tiers? La réponse à cette question est moins évidente. L'on pourrait envisager la situation où une alerte légitime portant sur un droit fondamental comme, par exemple, le droit à la vie ou à la santé, est méconnue, car le message manque de fiabilité du fait de la prime financière perçue par le lanceur d'alerte. Dans un tel cas, la prime aurait un effet négatif sur les droits fondamentaux.

La question empirique est donc soulevée de savoir si, de la fréquence de la présence d'une récompense financière, résulte la méconnaissance du caractère légitime d'une alerte portant sur les droits fondamentaux. Cette fréquence est-elle plus importante que le nombre d'alertes, portant elles aussi sur les droits fondamentaux, déclenchées et traitées grâce à la possibilité d'une récompense financière? Ces questions empiriques, ainsi que celles portant sur l'effet des récompenses financières sur le traitement des alertes par les agences publiques, restent à être étudiées.

Sur le plan purement théorique, il demeure difficile de répondre à la question des effets des récompenses financières sur les droits fondamentaux, car ces récompenses semblent avoir à la fois des effets bénéfiques et néfastes. En l'absence d'une étude empirique fiable, la meilleure leçon pour les juristes des deux côtés de l'Atlantique est peut-être d'hésiter avant de conclure que les récompenses financières des lanceurs d'alerte sont à adopter ou rejeter de manière universelle.

\section{NOTES}

1. Voir Vaughn Robert G., The Successes and Failures of Whistleblower Laws, Cheltenham, Edward Elgar Publishing Limited, 2012, p. 239.

2. Ibid. Voir également, pour une analyse générale du droit d'alerte américain, Modesitt Nancy M. et al., Whistleblowing: The Law of Retaliatory Discharge, Arlington, Bloomberg BNA, 2015.

3. 31 U.S.C. $\$ 3729$ et seq.

4. 15 U.S.C. $\S 78 \mathrm{u}-6$.

5. 26 U.S.C. $\S 7623$.

6. Pour une analyse des lois qui récompensent les lanceurs d'alerte dans divers pays tels le Royaume-Uni, la Hongrie, la Lituanie, le Pakistan, ou la République du Ghana, voir Faunce Tom et al., «Because they have evidence: globalizing financial incentives for corporate fraud 
whistleblowers ", in International Handbook on Whistleblowing Research, A.J. Brown et al. (dir.), Cheltenham, Edward Elgar Publishing Limited, 2014, p. 381-404.

7. Voir Conseil d'État, Le droit d'alerte : signaler, traiter, protéger, La Documentation française, 2016, p. 76.

8. Ibid.

9. La locution « qui tam » vient de la phrase latine « qui tam pro domino rege quam pro se ipso in hac parte sequitur", ce qui se traduit par "celui qui este en justice pour le compte de l'autorité publique (le roi) comme pour le sien propre ». Traduction d'Anquetil Alain, «Le rôle de l'alerte professionnelle face au déclin du bien public », Le Blog d'Éthique des Affaires, ESSCA École de Management, 19 janvier 2012. URL : http://www.essca.fr/blogs/ethique-des-affaires/ 2012/01/19/le-role-de-1_alerte-professionnelle-face-au-declin-du-bien-public/, consulté le 15 février 2016. Au regard de la référence faite au « roi », il n'est point étonnant d'apprendre que les États-Unis ont hérité ces lois qui tam de l'Angleterre, qui jouissait de ce genre de dispositif dès le $14^{\mathrm{e}}$ siècle. Pour une histoire des lois qui tam aux États-Unis et en Angleterre, voir Beck J. Randy, "The False Claims Act and the English Eradication of Qui Tam Legislation », in North Carolina Law Review, vol. 78, 2000, p. 539-642 ; Doyle Charles, Qui Tam: The False Claims Act and Related Federal Statutes, Rapport $\mathrm{n}^{\circ} \mathrm{R} 40785$, Washington, D.C., Congressional Research Service, 2009.

10. Voir, par exemple, 31 U.S.C. $\$ 3729$ et seq.

11. 35 U.S.C. app. $\$ 723$.

12. Voir Beck, «The False Claims Act and the English Eradication of Qui Tam Législation », op. cit., p. 567-568, qui décrit la 1318 Statute of York, une loi qui tam anglaise qui interdisait aux agents publics de vendre certaines marchandises, notamment les boissons alcoolisées.

13. Voir Doyle, Qui Tam : The False Claims Act and Related Federal Statutes, op. cit., p. 5.

14. Ibid.; voir également Beck, "The False Claims Act and the English Eradication of Qui Tam Legislation », op. cit., p. 555.

15. Doyle, Qui Tam : The False Claims Act and Related Federal Statutes, op. cit., p. 5.

16. 31 U.S.C. $\S 3729(\mathrm{a})(1)$.

17. Le lanceur d'alerte, devenu partie demanderesse dans une action qui tam, est connu sous le nom de « relator».

18. 31 U.S.C. $\S 3730(b)(1)$.

19. 31 U.S.C. $\S 3730(b)(2)$

20. Ibid.

21. Ibid. Le tribunal peut prolonger cette période sur demande motivée du procureur. Dans la plupart des actions qui tam, la période est prolongée, et le procureur général continue à enquêter pendant des mois ou des années avant de prendre sa décision de poursuivre, ou pas.

22. 31 U.S.C. $\$ 3730(b)(4)$. Le procureur général peut également demander au tribunal de rejeter la plainte, ce que le procureur ne fait que dans une petite minorité des cas.

23. 31 U.S.C. $§ 3730(d)(1)-(2)$. La part du lanceur d'alerte est réduite si les informations relatées par ce dernier ne sont pas complètement nouvelles, même si le lanceur d'alerte rajoute des éléments aux faits déjà connus. Dans ces cas, le lanceur d'alerte ne peut obtenir que 10 pour cent des dommages-intérêts, au maximum. Voir 31 U.S.C. § $3730(\mathrm{~d})(1)$. Le tribunal peut également diminuer la part du lanceur d'alerte si ce dernier a «organisé et initié les méfaits » («planned and initiated the violation »); si le lanceur d'alerte est condamné par une juridiction pénale pour le rôle qu'il a joué dans cette affaire, rien ne peut lui être octroyé. Voir 31 U.S.C. § 3730(d)(3).

24. United States Department of Justice, «Justice Department Announces Largest Health Care Fraud Settlement in its History ", Press Release, 2 septembre 2009. URL : http://www.justice.gov/ sites/default/files/usao-ma/legacy/2012/10/09/Pfizer \%20- \%20PR \%20 \%28Final \%29.pdf, consulté le 25 février 2016.

25. United-States Department of Justice, « GlaxoSmithKline to Plead Guilty and Pay \$ 3 Billion to Resolve Fraud Allegations and Failure to Report Safety Data », Press Release, 2 juillet 2012. URL : 
http://www.justice.gov/opa/pr/glaxosmithkline-plead-guilty-and-pay-3-billion-resolve-fraudallegations-and-failure-report, consulté le 25 février 2016.

26. Loftus Peter, « Whistleblower's Long Journey », in The Wall Street Journal [En ligne], 29 octobre 2010. URL: http://www.wsj.com/articles/SB10001424052702303443904575578713255698500, consulté le 25 février 2016.

27. Selon Miceli, Near et Dworkin, qui figurent entre les chercheurs américains les plus éminents en matière de lancement d'alerte, le False Claims Act est la loi qui a le mieux fonctionné pour inciter et protéger les lanceurs d'alerte. Miceli Marcia P., Near Janet P., Dworkin Terry Morehead, Whistleblowing in Organizations, New York, Routledge, 2008, p. 176.

28. United States Department of Justice, «Justice Department Recovers Nearly \$ 6 Billion from False Claims Act Cases in Fiscal Year 2014 ", Press Release, 20 novembre 2014. URL : http:// www.justice.gov/opa/pr/justice-department-recovers-nearly-6-billion-false-claims-act-casesfiscal-year-2014, consulté le 25 février 2016.

29. Meyer Jack A., Fighting Medicare \& Medicaid Fraud: The Return on Investment from False Claims Act Partnerships, Taxpayers Against Fraud Education Fund, octobre 2013. URL : https://www.taf.org/ TAF-ROI-report-October-2013.pdf, consulté le 4 juillet 2016.

30. 35 U.S.C. $\S 292$.

31. 25 U.S.C. $\S 201$.

32. Voir Doyle, Qui Tam : The False Claims Act and Related Federal Statutes, op. cit., p. 20-23.

33. Taxpayers Against Fraud Education Fund, "States With False Claims Acts ", http:// www.taf.org/states-false-claims-acts, consulté le 11 mars 2015.

34. Robert Vaughn appelle ces lois des «bounty laws». Vaughn, The Successes and Failures of Whistleblower Laws, op. cit., p. 137.

35. 15 U.S.C. § 78u-6(a), (b).

36. L'allocation exacte dépend de plusieurs facteurs, comme la pertinence des informations, l'utilisation préalable des voies de recours interne, ou les délais dans la transmission des informations. 15 U.S.C. § 78u-6(c)(1) ; 17 C.F.R. § 240.21F-6.

37. 15 U.S.C. $\S 78 u-6(h)(2)$.

38. 15 U.S.C. $\S 78 \mathrm{u}-6(\mathrm{~h})(1)$.

39. U.S. Securities and Exchange Commission, « 2015 Annual Report to Congress on the DoddFrank Whistleblower Program», 16 novembre 2015, p. 1. URL : https://www.sec.gov/ whistleblower/reportspubs/annual-reports/owb-annual-report-2015.pdf, consulté le 26 février 2016.

40. Ibid., p. 10.

41. Voir Order Determining Award Claim, SEC Rel. No. 73174, File No. 2014-10, 22 septembre 2014. URL : http://www.sec.gov/rules/other/2014/34-73174.pdf, consulté le 26 février 2016. À la différence des récompenses financières, la protection contre les représailles prévue par la loi Dodd-Frank ne s'applique pas aux personnes étrangères travaillant hors des États-Unis sous contrat avec un employeur non-américain. Voir ibid., p. 2 ; voir également Liu v. Siemens AG, 763 F. 3d 175 (2d Cir. 2014), un arrêt dans lequel la Cour d'appel des États-Unis pour le deuxième circuit jugea que le dispositif de la loi Dodd-Frank interdisant toute discrimination contre les lanceurs d'alerte ne s'appliquait pas de manière extraterritoriale. Pour une discussion en langue française de cet arrêt, voir Zekkouti Naguin B., «L'extraterritorialité des règles boursières américaines à l'ère post-Morrison : une double confirmation qui soulève des questions ", in Bull. Joly Bourse, $\mathrm{n}^{\circ} 1$, 31 janvier 2015.

42. Voir U.S. Securities and Exchange Commission, « 2015 Annual Report to Congress on the Dodd-Frank Whistleblower Program », op. cit., p. 1.

43. Ibid.

44. 26 U.S.C. $\$ 7623$. 
45. Birkenfeld a également été condamné à 40 mois de prison ferme pour avoir participé à l'évasion dénoncée. Voir Kocieniewski David, « Whistle-Blower Awarded \$ 104 Million by I.R.S ", in The New York Times [En ligne], 11 septembre 2012. URL : http://www.nytimes.com/2012/09/12/ business/whistle-blower-awarded-104-million-by-irs.html, consulté le 4 juillet 2016.

46. Pangloss est le précepteur de Candide, le personnage principal du conte philosophique « Candide ou l'Optimisme ", écrit par le philosophe français Voltaire et publié en janvier 1759.

47. Voir par exemple Callahan Elletta Sangrey, Dworkin Terry Morehead, «Do Good and Get Rich : Financial Incentives for Whistleblowing and the False Claims Act », in Villanova Law Review, vol. 37, 1992, p. 273-336 ; Bishara Norman D. et. al., « The Mouth of Truth », in New York University Journal of Law and Business, vol. 10, 2013, p. 37-114; Bucy Pamela H., «Private Justice », in Southern California Law Review, vol. 76, 2002, p. 53 ; Helmer James B., Jr., « How Great Is Thy Bounty: Relator's Share Calculations Pursuant to the False Claims Act», in University of Cincinnati Law Review, vol. 68, 2000, p. 737-761 ; Fisch Jill E., « Class Action Reform, Qui Tam, and the Role of the Plaintiff », in Journal of Law and Contemporary Problems, vol. 60, 1997, p. 167-202.

48. Callahan et Dworkin, "Do Good and Get Rich : Financial Incentives for Whistleblowing and the False Claims Act », op. cit., p. 283-324 ; Bishara, "The Mouth of Truth", op. cit., p. 60-61 ; Bucy, « Private Justice », op. cit., p. 54.

49. Bucy, «Private Justice », op. cit., p. 59-62 ; Fisch, « Class Action Reform, Qui Tam, and the Role of the Plaintiff », op. cit., p. 196.

50. Il s'agit de Frank Camps et Karen Silkwood. Government Accountability Project, «A Timeline of US Whistleblowers ", https://www.whistleblower.org/timeline-us-whistleblowers, consulté le 1 juillet 2016.

51. Bucy, « Private Justice », op. cit., p. 59-62.

52. Ibid., p. 32-33.

53. Fowler Renn C., L'Heureux Robert D., A Guide to the Whistleblower Protection Act \& Related Litigation, Arlington (Virginie États-Unis), Dewey Publications, 2001, p. 2.

54. Il est pertinent de regarder l'expérience, en France, de Tracfin, le service de renseignement rattaché au Ministère des Finances et des Comptes publics, chargé de recueillir les déclarations de soupçons de la part de certains professionnels relatives à des infractions de blanchiment de capitaux ou de financement du terrorisme. En 2014, ce service a reçu plus de 38000 informations, soit une hausse de $33 \%$ sur l'année précédente. Tracfin n'a pourtant pu mener que 9782 enquêtes au cours de l'année 2014 , soit une hausse de 5,5\%, par rapport à l'année précédente, et plus de la moitié de ces enquêtes étaient liées aux informations reçues au cours de l'année 2013 ou des années antérieures. Voir Tracfin, «Rapport annuel d'activité Tracfin 2014 », p. 7. URL : http://www.economie.gouv.fr/files/tracfin_2014.pdf, consulté le 7 mars 2016.

55. Devine Thomas M., Blowing the Whistle on Retaliation: Accounts of Current and Former Federal Agency Whistleblowers, Testimony before U.S. Senate Committee on Homeland Security \& Governmental Affairs, 11 juin 2015. URL : www.hsgac.senate.gov/hearings/blowing-the-whistleon-retaliation-accounts-of-current-and-former-federal-agency-whistleblowers, consulté le 16 juin 2015.

56. United-States Department of Justice, «Justice Department Recovers Nearly \$ 6 Billion from False Claims Act Cases in Fiscal Year 2014 », op. cit. ; Meyer, Fighting Medicare \& Medicaid Fraud: The Return on Investment from False Claims Act Partnerships, op. cit., p. 6.

57. Le rapport annuel 2015 de la SEC indique qu'elle a versé environ USD 54 millions aux lanceurs d'alerte depuis l'entrée en vigueur du Dodd Frank Act en 2011. U.S. Securities and Exchange Commission, « 2015 Annual Report to Congress on the Dodd-Frank Whistleblower Program », op. cit., p. 1. Les récompenses prévues par Dodd Frank étant de 10 à 30 pour cent des fonds récupérés, la fourchette des fonds récupérés semble se situer entre un maximum de 540 millions (tout lanceur d'alerte ayant reçu une prime de 10 pour cent) et un minimum de 180 millions de dollars (tout lanceur d'alerte ayant reçu une prime de 30 pour cent). 
58. Voir par exemple Beck, "The False Claims Act and the English Eradication of Qui Tam Legislation », op. cit., p. 638.

59. Meyer, Fighting Medicare \& Medicaid Fraud: The Return on Investment from False Claims Act Partnerships, op. cit., p. 1.

60. Fisch, «Class Action Reform, Qui Tam, and the Role of the Plaintiff », op. cit., p. 196-197.

61. Voir Conseil d'État, Le droit d'alerte : signaler, traiter, protéger, op. cit., p. 76.

62. Voir, par exemple, Council of Europe, Protection of Whistleblowers: Recommendation CM/ $\operatorname{Rec}(2014) 7$ adopted by the Committee of Ministers of the Council of Europe on 30 April 2014 and explanatory memorandum, 30 April 2014, Principle 10 ; Conseil d'État, Le droit d'alerte : signaler, traiter, protéger, op. cit., p. 7 ; Commission Nationale de l'Informatique et des Libertés, Autorisation unique $\mathrm{n}^{\circ} \mathrm{AU}-004$, Version consolidée au 15 février 2016. URL : http://www.cnil.fr/sites/default/files/ atoms/files/au-004-consolidee.pdf, consulté le 4 juillet 2016.

63. Rich Michael, «Prosecutorial Indiscretion: Encouraging the Department of Justice to Rein in Out-of-Control Qui Tam Litigation Under the Civil False Claims Act », in University of Cincinnati Law Review, vol. 76, 2008, p. 1263-1264 ; Elameto Sean, "Guarding the Guardians : Accountability in Qui Tam Litigation Under the Civil False Claims Act », in Public Contract Law Journal, vol. 41, 2012, p. 813-854; Beck, "The False Claims Act and the English Eradication of Qui Tam Legislation", op. cit., p. 622-627; Kovacic William E., "Whistleblower Bounty Lawsuits As Monitoring Devices in Government Contracting », in Loyola of Los Angeles Law Review, vol. 29, 1996, p. 1831 1832; Castleton Todd B., «Compounding Fraud: The Costs of Acquiring Relator Information Under the False Claims Act and the 1993 Amendments to Federal Rules of Civil Procedure ", in George Mason Law Review, vol. 4, 1996, p. 327-368.

64. Kovacic William E., «The Civil False Claims Act As A Deterrent to Participation in Government Procurement Markets », in Supreme Court Economic Review, vol. 6, 1998, p. 225.

65. Ibid.

66. Voir Conseil d'État, Le droit d'alerte: signaler, traiter, protéger, op. cit., p. 73 ; U.S. ex rel. Miller v. Bill Harbert Int'l Const., Inc., 505 F. Supp. 2d 20, 28 (D.D.C. 2007) ; U.S. ex rel. Stephens v. Prabhu, No. CV-S-92-653, 1994 WL 761237, at *1 (D. Nev. Dec. 14, 1994).

67. La False Claims Act autorise le tribunal à enjoindre au lanceur d'alerte de payer les frais et honoraires de la partie défenderesse si l'action était «manifestement frivole, manifestement vexatoire, ou intentée principalement avec intention de nuire». Voir 31 U.S.C. $§ 3730(d)(4)$ (« clearly frivolous, clearly vexatious, or brought primarily for the purposes of harassment »).

68. 637 F.3d 1047 (9th Cir. 2011).

69. Ibid., p. 1062.

70. Ibid.

71. Voir United-Nations Office on Drugs and Crime, Resource Guide on Good Practices in the Protection of Reporting Persons, août 2015, p. 26. URL: http://www.unodc.org/documents/ corruption/Publications/2015/15-04741_Person_Guide_eBook.pdf, consulté le 29 juin 2016.

72. Callahan et Dworkin, « Do Good and Get Rich : Financial Incentives for Whistleblowing and the False Claims Act », op. cit., p. 334-335.

73. Ibid.

74. Sur ce point, voir Miethe Terance D., Whistleblowing at Work: Tough Choices in Exposing Fraud, Waste, and Abuse on the Job, Boulder (Colorado États-Unis), Westview Press, 1999, p. 27-28.

75. Conseil d'État, Le droit d'alerte : signaler, traiter, protéger, op. cit., p. 76. 


\section{RÉSUMÉS}

Le droit américain prévoit plusieurs dispositifs de récompense financière pour les lanceurs d'alerte. Ces dispositifs prennent deux formes principales : des lois qui tam, qui permettent au lanceur d'alerte de porter plainte au nom du gouvernement, et des lois compensatoires dans le sens strict du terme, qui octroient une somme d'argent à un lanceur d'alerte si les informations qu'il a fournies ont conduit à un recouvrement de fonds par l'État. Ces lois semblent avoir la possibilité à la fois de protéger et de porter atteinte aux droits fondamentaux des personnes mises en cause par l'alerte, des tierces personnes, et du lanceur d'alerte lui-même.

There are a number of American statutes containing whistleblower reward provisions. These statutes take two principal forms: qui tam laws, which allow the whistleblower to pursue an action on behalf of the government, and reward laws in the strict sense of the term, which merely promise whistleblowers a certain sum of money when their tips lead to a successful enforcement action. Reward laws appear to have the possibility of both protecting and undermining fundamental rights, including those of the target of the whistleblower's disclosure, third persons, and the whistleblower.

\section{INDEX}

Keywords : whistleblowers, financial rewards, whistleblower incentives, « bounty laws », False Claims Act, qui tam, Dodd-Frank, american law, fundamental rights

Mots-clés : lanceurs d'alerte, récompenses financières, incitations à l'alerte, « bounty laws », False Claims Act, qui tam, Dodd-Frank, droit américain, droits fondamentaux

\section{AUTEUR}

\section{JOHANNA SCHWARTZ MIRALLES}

Doctorante en droit privé à l'université d'Aix-Marseille, Centre de droit économique (EA 4224) 